\title{
Inhibition of very late antigen-4 and leukocyte function-associated antigen-1 in experimental autoimmune uveoretinitis.
}

Purpose: To investigate whether experimental autoimmune uveoretinitis (EAU) can be inhibited by treatment with blocking Abs specific for very late antigen-4 (VLA-4) and leukocyte function-associated antigen-1 (LFA-1).

Methods: B10.RIII mice were immunized with interphotoreceptor retinoid binding protein (IRBP) peptide 161-180 in complete Freund's adjuvant together with pertussis toxin. To investigate the involvement of VLA-4 and LFA-1 in EAU, expression of these molecules in the retina of EAU-developing mice was evaluated by immunohistochemistry. To examine whether EAU can be inhibited by blocking of these molecules, anti-VLA-4, anti-LFA-1 or control antibody (Ab) was intraperitoneally injected every other day from 5 and 13 days after immunization (effector phase). Fourteen days and 28 days after immunization, the eyes and spleens were harvested. The eyes were used for histologic analysis and cytokine mRNA expression analysis. The spleens were used for Ag-recall cytokine production assays and intracellular cytokine assays.

Results: Expression of VLA-4 and LFA-1 in the eye was upregulated by EAU induction. Effector phase treatment with anti-LFA-1 Ab, but not with anti-VLA-4 Ab, significantly reduced the severity of EAU. Treatment with both Abs significantly and more profoundly suppressed the severity of EAU. The levels of cytokine mRNA expression in the eye were significantly suppressed by treatment with anti-VLA-4 and anti-LFA-1 Abs. In contrast, splenocyte cytokine production and intracellular IFN- $\gamma+$ 
and IL-17A+ cytokine profile of CD4+ T cells were significantly upregulated with both Abs. However, discontinuation of the Ab treatment abrogated the suppressive effects on EAU severity and cytokine mRNA expression in the eye together with upregulation of splenocyte cytokine production and intracellular IFN- $\gamma+$ and IL-17A+cytokine profile of CD4+ T cells.

Conclusion: VLA-4 and LFA-1 appear to play a significant role in the development of EAU during the effector phase. Treatment with Abs specific for these molecules can be a therapeutic candidate for patients with uveitis, however, Ab treatment is needed to be continued. 